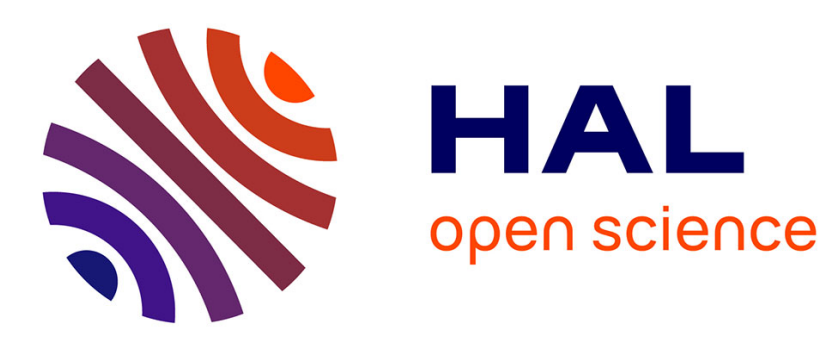

\title{
Gaz de schiste en Europe: le mirage des emplois
}

Stéphane Goutte, Thomas Porcher

\section{To cite this version:}

Stéphane Goutte, Thomas Porcher. Gaz de schiste en Europe: le mirage des emplois. Gaz naturel, la nouvelle donne, pp.55-60, 2016. halshs-02615592

\section{HAL Id: halshs-02615592 \\ https://shs.hal.science/halshs-02615592}

Submitted on 29 May 2020

HAL is a multi-disciplinary open access archive for the deposit and dissemination of scientific research documents, whether they are published or not. The documents may come from teaching and research institutions in France or abroad, or from public or private research centers.
L'archive ouverte pluridisciplinaire HAL, est destinée au dépôt et à la diffusion de documents scientifiques de niveau recherche, publiés ou non, émanant des établissements d'enseignement et de recherche français ou étrangers, des laboratoires publics ou privés. 


\title{
Gaz de schiste en Europe : le mirage des emplois
}

\author{
Thomas Porcher et Stéphane Goutte
}

Dans une étude publiée en 2011, l’IHS avance le chiffre de 601348 emplois créés (miniers et non miniers) aux États-Unis grâce à l'exploitation du gaz de schiste. Selon l'institut, 57381 seraient des emplois miniers et 543967 des emplois non miniers (transports, services...) ${ }^{1}$. Chaque emploi minier créé a donc entraîné la création de plus de neuf emplois non miniers. Un multiplicateur d'emplois très élevé qui en ferait un des secteurs les plus créateurs d'emplois aux États-Unis, devant la construction. L'objet de ce chapitre est de savoir si ce multiplicateur «emplois miniers/emplois non miniers » est transposable au cas de l'Europe.

\section{Les emplois non miniers sont-ils uniquement dus à la production de gaz de schiste?}

Les tenants du gaz de schiste reconnaissent que les emplois miniers ont toujours été faibles dans l'industrie pétrolière et gazière, mais avancent que les emplois non miniers peuvent être très élevés. Le problème est que la plupart de ces emplois ne dépendent pas uniquement de l'exploitation du gaz de schiste, mais aussi de celle du gaz conventionnel, du gaz de couche ou du tight gas. Par exemple, les infrastructures de distribution ou la présence d'une industrie de services très développée aux États-Unis ne dépendent pas uniquement de l'exploitation des gaz de schiste (autour de 50000 puits $^{2}$ ), mais des 500000 puits de gaz actuellement en activité. Idem pour la baisse des prix du gaz américain : elle ne peut pas être entièrement attribuée au gaz de schiste alors qu'il y a eu plus de 27000 nouveaux puits de gaz en activité entre 2010 et 2011 et 108000 entre 2004 et 2011. À cela il faut ajouter la baisse de la demande avec la crise économique (entraînant une double pression à la baisse sur les prix). La production de gaz de schiste a certes contribué à la baisse des prix du gaz et à la création d'emplois, mais il est

\footnotetext{
${ }^{1}$ «The Economic and Employment Contributions of Shale Gas in the United States », IHS Global Insight, décembre 2011. Disponible en ligne : http://energyindepth.org/wp-content/uploads/2011/12/Shale-GasEconomic-Impact-Dec-2011_EMB1.pdf

${ }^{2}$ Chiffre avancé par Bruno Courme (Total) au débat national sur la transition énergétique (DNTE) au ministère de l'Écologie, 3 juillet 2013, Paris.
} 
faux de lui attribuer l'ensemble de ces résultats. Même conclusion pour les pétroles de schiste : leur production a contribué à exercer une pression à la baisse sur les prix du pétrole, mais on ne peut pas honnêtement avancer qu'elle en soit l'unique cause - le renchérissement du dollar et la baisse de la croissance mondiale sont d'autres facteurs à prendre en compte. De même attribuer les emplois créés par la baisse du prix du pétrole à la seule production de pétrole de schiste n'est pas économiquement honnête.

\section{Le calcul des multiplicateurs emplois miniers/emplois non miniers}

Le calcul de ces emplois non miniers est un exercice difficile et largement contestable. Néanmoins, des recherches se sont penchées sur le calcul du multiplicateur entre la création d'emplois miniers et non miniers dans le cas des États-Unis.

Dans un article scientifique, Jeremy G. Weber, en se fondant sur 362 comtés américains, montre que chaque milliard de $\mathrm{m}^{3}$ de production de gaz crée 18,5 emplois dans le comté de production $^{3}$. Sur ces 18,5 emplois, 7,5 sont des emplois dans le secteur minier et 11 sont non miniers. Chaque emploi créé dans le secteur minier engendre donc la création d' 1,4 emploi dans d'autres secteurs. Le multiplicateur d'emploi est donc beaucoup plus faible dans l'analyse de J. G. Weber.

En fonction des études, le multiplicateur est donc compris entre 1,4 et 9. IHS rappelle que la force du multiplicateur dépend principalement du fait que l'ensemble des fournisseurs de l'industrie du gaz de schiste (matériaux, services, produits métalliques...) sont américains et de surcroît des leaders mondiaux dans ces secteurs. Contrairement à d'autres industries ayant des fournisseurs à l'étranger, les sommes investies dans l'industrie du gaz de schiste restent donc aux États-Unis, ce qui explique la valeur élevée du multiplicateur. Pour J. G. Weber, le multiplicateur d'IHS est valable dans les quatre États américains qui ont la chaine de développement du gaz de schiste la plus développée au monde. Le multiplicateur d'IHS peut donc être considéré comme une borne supérieure, mais ne peut pas être appliqué à l'ensemble des États-Unis et encore moins au reste du monde.

On peut donc considérer qu'en fonction de l'État où l'on se trouve, le multiplicateur d'emplois aux États-Unis est compris entre 1,4 et 9. Or, nous savons que la valeur du multiplicateur dépend principalement du niveau de développement de l'industrie gazière, de l'offre de services qui va avec et de la baisse des prix de l'énergie. Dans le secteur pétrolier et

\footnotetext{
${ }^{3}$ Jeremy G. Weber, « A Decade of Natural Gas Development. The Makings of a Resource Curse ? , Resource and Energy Economics, août 2014, vol. 37, p. 168-183.
} 
gazier, les États-Unis ont un quasi-monopole des moyens, et si on y ajoute le fait que la baisse des prix sera limitée en Europe (de 10 à $20 \%$ à l'horizon 2035 selon les études les plus optimistes), on peut donc conclure que le multiplicateur d'emploi en Europe sera probablement beaucoup plus faible qu'aux États-Unis.

La plupart des estimations en Europe prévoit pourtant un multiplicateur plus élevé. Des scénarios de développement du gaz de schiste en Europe entre 2020 et 2050 avancent la création de 0,5 à 1,1 million d'emplois et le forage de 23000 à 50000 puits $^{4}$. Sachant que le rapport emploi minier/puits est proche d'un emploi par puits (57 381 emplois miniers pour 50000 puits forés aux États-Unis), les scénarios européens n'ont plus un multiplicateur à 9 (borne supérieure des États-Unis), mais à 21. Par conséquent, soit les prévisions sont largement surévaluées $^{5}$, soit il va falloir forer plus de puits.

Nous savons que le multiplicateur d'emploi minier/non minier en Europe est probablement bien plus faible que la borne inférieure américaine $(1,4)$, mais même en considérant qu'elle est équivalente, les 23000 à 50000 puits annoncés dans les scénarios précédents amèneraient à la création de 55000 à 120000 emplois dans toute l'Europe à l'horizon 2035.

En reprenant les ressources estimatives des gaz de schiste en France par rapport à l'Europe et en se fondant sur les hypothèses de puits des deux scénarios, on peut estimer le nombre de puits en France. Sachant que la France représente $29 \%$ des réserves estimées européennes ${ }^{6}$, le nombre de puits nécessaires se situe entre 6670 et 14 500. En refaisant les calculs précédents, on arrive à des créations d'emplois entre 16008 et 34800 (d'ici à 2035). À cela, il faut ajouter que les réserves de gaz de schiste étant parfois situées en France dans des régions touristiques, il pourrait y avoir des destructions d'emplois dans le secteur du tourisme ce qui ne garantit pas une création d'emplois nets.

$\mathrm{Au}$ final, même en utilisant un multiplicateur d'emplois américain, les estimations des créations d'emplois grâce à l'exploitation du gaz de schiste s'avèrent beaucoup trop faibles pour répondre au problème du chômage de masse en France et en Europe.

\footnotetext{
${ }^{4}$ Philippe Charlez, « La révolution américaine des énergies non conventionnelles », Risques, septembre 2014, $\mathrm{n}^{\circ} 99$.

${ }^{5}$ Dans un article scientifique publié dans la revue Energy Economics, J. G. Weber montre que les estimations des créations d'emplois avant exploitations avaient été surévaluées.

${ }^{6}$ Selon l'US Energy Information Administration, les ressources estimatives de gaz pour la France sont de

23 Gbep et de 80 Gbep pour l'Europe.
} 
